\title{
RepetitiveTMS suggests a role of the human dorsal premotor cortex in action prediction
}

\author{
Waltraud Stadler ${ }^{1,2}$ *, Derek V. M. Ott ${ }^{1}$, Anne Springer ${ }^{1,3}$, Ricarda I. Schubotz ${ }^{4}$, Simone Schütz-Bosbach ${ }^{5}$ and \\ Wolfgang Prinz ${ }^{1}$
}

${ }^{1}$ Department of Psychology, Max Planck Institute for Human Cognitive and Brain Sciences, Leipzig, Germany

${ }^{2}$ Human Movement Science, Department of Sports and Health Science, Technische Universität München, Munich, Germany

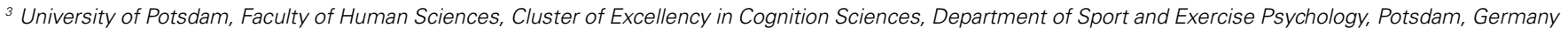

${ }^{4}$ Motor Cognition Group, Max Planck Institute for Neurological Research, Cologne, Germany

5 Junior Research Group "Body and Self," Max Planck Institute for Human Cognitive and Brain Sciences, Leipzig, Germany

Edited by:

Francisco Barcelo, University of Illes

Balears, Spain

\section{Reviewed by:}

Lutz Jäncke, University of Zurich,

Switzerland

Edward De Haan, Faculty of Social and Behavioural Sciences,

Netherlands

\section{*Correspondence:}

Waltraud Stadler, Human Movement

Science, Department of Sports and

Health Science, Technische

Universität München, Campus D,

Georg-Brauchle-Ring 60-62, 80992

München, Germany.

e-mail:waltraud.stadler@tum.de
Predicting the actions of other individuals is crucial for our daily interactions. Recent evidence suggests that the prediction of object-directed arm and full-body actions employs the dorsal premotor cortex (PMd). Thus, the neural substrate involved in action control may also be essential for action prediction. Here, we aimed to address this issue and hypothesized that disrupting the PMd impairs action prediction. Using fMRI-guided coil navigation, rTMS (five pulses, $10 \mathrm{~Hz}$ ) was applied over the left PMd and over the vertex (control region) while participants observed everyday actions in video clips that were transiently occluded for $1 \mathrm{~s}$. The participants detected manipulations in the time course of occluded actions, which required them to internally predict the actions during occlusion. To differentiate between functional roles that the PMd could play in prediction, rTMS was either delivered at occluder-onset (TMS-early), affecting the initiation of action prediction, or $300 \mathrm{~ms}$ later during occlusion (TMS-late), affecting the maintenance of an ongoing prediction. TMS-early over the left PMd produced more prediction errors than TMS-early over the vertex. TMS-late had no effect on prediction performance, suggesting that the left PMd might be involved particularly during the initiation of internally guided action prediction but may play a subordinate role in maintaining ongoing prediction. These findings open a new perspective on the role of the left PMd in action prediction which is in line with its functions in action control and in cognitive tasks. In the discussion, the relevance of the left PMd for integrating external action parameters with the observer's motor repertoire is emphasized. Overall, the results are in line with the notion that premotor functions are employed in both action control and action observation.

Keywords: action observation, prediction, occlusion, premotor, PMd, transcranial magnetic stimulation

\section{INTRODUCTION}

An increasing amount of evidence points to the relevance of the premotor cortex for predicting events in the external world (see Schubotz, 2007, for a review). The premotor cortex plays a central role in action planning and forms a core part of the network that is activated when an action is observed or imagined (Grèzes and Decety, 2001). It has been suggested that the involvement of the motor system during action observation mainly serves predictive purposes (Wilson and Knoblich, 2005; Schütz-Bosbach and Prinz, 2007; Urgesi et al., 2010). We obtained corresponding evidence in a study using functional magnetic resonance images (fMRI; Stadler et al., 2011), which showed that the left dorsal premotor cortex (PMd) and the left pre-supplementary motor area (pre-SMA) were involved in the prediction of actions that were transiently occluded from view. The particular relevance of the left PMd for internal action prediction can be explained by the type of actions used in the experiment: participants predicted objectdirected (i.e., transitive) arm and full-body actions performed in a naturalistic environment by a right-handed actress.
In the present study, we tested whether the left PMd has a causal significance for internal action prediction. We hypothesized that disturbing this area by means of rTMS impairs the prediction of transiently occluded actions.

Other studies using TMS to investigate premotor cortex involvement in action observation focused on the pars opercularis of the inferior frontal gyrus (IFG), which has been suggested to belong to the posteriorly adjacent ventral premotor cortex (PMv). Setting transient lesions in this area seems to disrupt sensorimotor transformations of action-related visual information (Urgesi et al., 2007a) and leads to performance deficits only when observers are required to access action representations that are in their own action repertoire (Pobric and Hamilton, 2006; Avenanti et al., 2007; Urgesi et al., 2007b), for example, when discriminating between possible but not between impossible hand configurations (Candidi et al., 2008).

So far, TMS has not been used to study the involvement of premotor areas and particularly the PMd during the active prediction of observed actions. Studies on motor control indicate that TMS 
over this area affects different aspects of motor cognition as compared to PMv. Davare et al. (2006) found that during grasping and subsequent lifting of an object, rTMS over the PMv affects the grip configuration while stimulation over the PMd selectively disrupts the timing of the lifting phase. This is in line with the functional distinction between PMv and PMd that is established in literature (see Tomassini et al., 2007 for a dissociation in humans and an overview of findings in the monkey). The PMv is associated with the control of fingers in coordination with the surface of the grasped object. The PMd is involved in the control of movements that integrate a higher number of joint trajectories such as reaching for objects in space (Pesaran et al., 2006; Beurze et al., 2007). Furthermore, the PMd has been shown to contribute to learned associations of actions with arbitrary external events (Rushworth et al., 2003). Neurons in the PMd of monkeys were found to represent the expected external effects of performed and observed actions in a task in which markers lighted up when touched with a cursor (Cisek and Kalaska, 2004). Correspondingly, TMS over the human PMd disrupts the prediction of forces when different weights are lifted (Chouinard et al., 2005) and prolongs reaction times (RT) in choice reaction time tasks (Schluter et al., 1998; Johansen-Berg et al., 2002) when actions are contingent on arbitrary visual cues.

Moreover, the PMd is involved in more abstract cognitive tasks that are not directly related to action but require spatial transformations, such as serial prediction (Schubotz and von Cramon, 2001; Schubotz et al., 2003), the generation of number sequences from memory (Abe et al., 2007) and mental rotation (Lamm et al., 2001; Windischberger et al., 2003). Mental rotation is a task that has many functional commonalities with action prediction. Both tasks require an internally guided transformation of visual (or visuospatial) information (see Schubotz, 2007). Interestingly, TMS over the PMd was shown to disrupt mental rotation (Tanaka et al., 2005; Oshio et al., 2010) and mental rotation can induce corticospinal excitation as observed in motor-evoked potentials measured in hand muscles (Bode et al., 2007; Eisenegger et al., 2007).

Assuming that the internal prediction of observed actions employs similar PMd functions as those involved in the generation of overt action, we expected that interference with rTMS impairs action prediction performance. We presented everyday actions in video clips and used a task that required participants to predict action sequences during transient occlusions lasting for $1 \mathrm{~s}$ (Figure 1A). Immediately after an occlusion, the action continued either with correct or manipulated timing. Manipulating the time course of the actions resulted in continuations that either lagged behind or were too advanced. The participants indicated whether the timing was correct or not by pressing one of two response buttons. Repetitive TMS at $10 \mathrm{~Hz}$ was applied over the left PMd and in a control session over the vertex region (VX). To guide rTMS application to each individual's prediction related "hot spot" in the PMd and to the VX region that was not related to prediction, previously acquired fMRI contrast images were used (Figure 1B). Repetitive TMS pulse trains lasted for $400 \mathrm{~ms}$ and were applied either at occluder-onset (TMS-early) or with a delay of $300 \mathrm{~ms}$ after occluder-onset (TMS-late). This variation of rTMS onsets allowed us to differentiate between the effects of PMd stimulation during early periods of internal prediction (i.e., initiating action prediction) and effects in later prediction periods (i.e., driving and maintaining ongoing action prediction). Applying TMS over the premotor cortex was shown to condition the excitability of the primary motor cortex (M1; Civardi et al., 2001; Rizzo et al., 2004). In order to avoid an influence on motor responses, we asked the participants to respond with the ipsilateral left hand. The electromyographic (EMG) activity was monitored and recorded during the entire TMS experiment and was compared between the stimulation sites.

\section{MATERIALS AND METHODS PARTICIPANTS}

Twelve right-handed (above 80\% according to Edinburgh Inventory; Oldfield, 1971) females, aged between 20 and 32 years (mean $=25 \pm 3.3)$ participated in the experiment. In accordance with the declaration of Helsinki, participants received information regarding effects of rTMS and related risks before signing an informed consent agreement. The experimental protocol was approved by the Ethics Committee of the University of Leipzig. None of the participants had neurological, psychiatric, or other medical problems or any contraindication to rTMS (Wassermann, 1998). One participant decided to withdraw from the experiment after the first session.

\section{STIMULI AND TASK}

Eight different video clips (mean duration: $37.8 \pm 11.2 \mathrm{~s}$, range: $11-52.5 \mathrm{~s}$ ) were presented in random order, each showing a female agent performing a well-known everyday action in a naturalistic setting (changing the bed sheets, filling the dishwasher, putting up a poster, setting the table, hanging up laundry, preparing a salad, making coffee, watering a plant). The participants were seated at a distance of $112 \mathrm{~cm}$ from the monitor. At a screen resolution of $1024 \times 768$ pixels, the agent in the video covered a visual angle of $5.76^{\circ}$. The clips were repeatedly occluded with a dark rectangle covering the whole display. All occlusions lasted for $1 \mathrm{~s}$. Each video clip was occluded one to four times (average $2.5 \pm 0.7$ ) and was presented six times using different occluder-onset positions in order to exclude possible learning effects due to repetition. Occlusions were placed at action sequences that were rated as sufficiently predictable on the basis of pilot experiments. In accordance with the functional selectivity of the PMd (Kalaska et al., 1997; Hoshi and Tanji, 2000; Raos et al., 2003; Beurze et al., 2007; Filimon et al., 2007), occlusions covered sequences of higher integrated limb and full-body actions that were extended in space, such as reaching for objects, object transport in space, or full-body translational movement. The interval between occlusions varied randomly between 7.5 and $20.5 \mathrm{~s}$ (mean $13.5 \pm 3.6$ ). After each occlusion, participants indicated whether the time course of the action was coherent or had been manipulated during the occlusion. In the coherent timing conditions, the action continuation corresponded to the occluder duration (i.e., the action continued at a frame occurring $1 \mathrm{~s}$ after the last visible frame before occlusion). In the incoherent timing conditions, the action either continued too soon (continuation at $0.5 \mathrm{~s}$ instead of $1 \mathrm{~s}$ after the last visible frame) or belated (continuation at $3 \mathrm{~s}$ instead of $1 \mathrm{~s}$ after the last visible frame). The asymmetry between time shifts in 


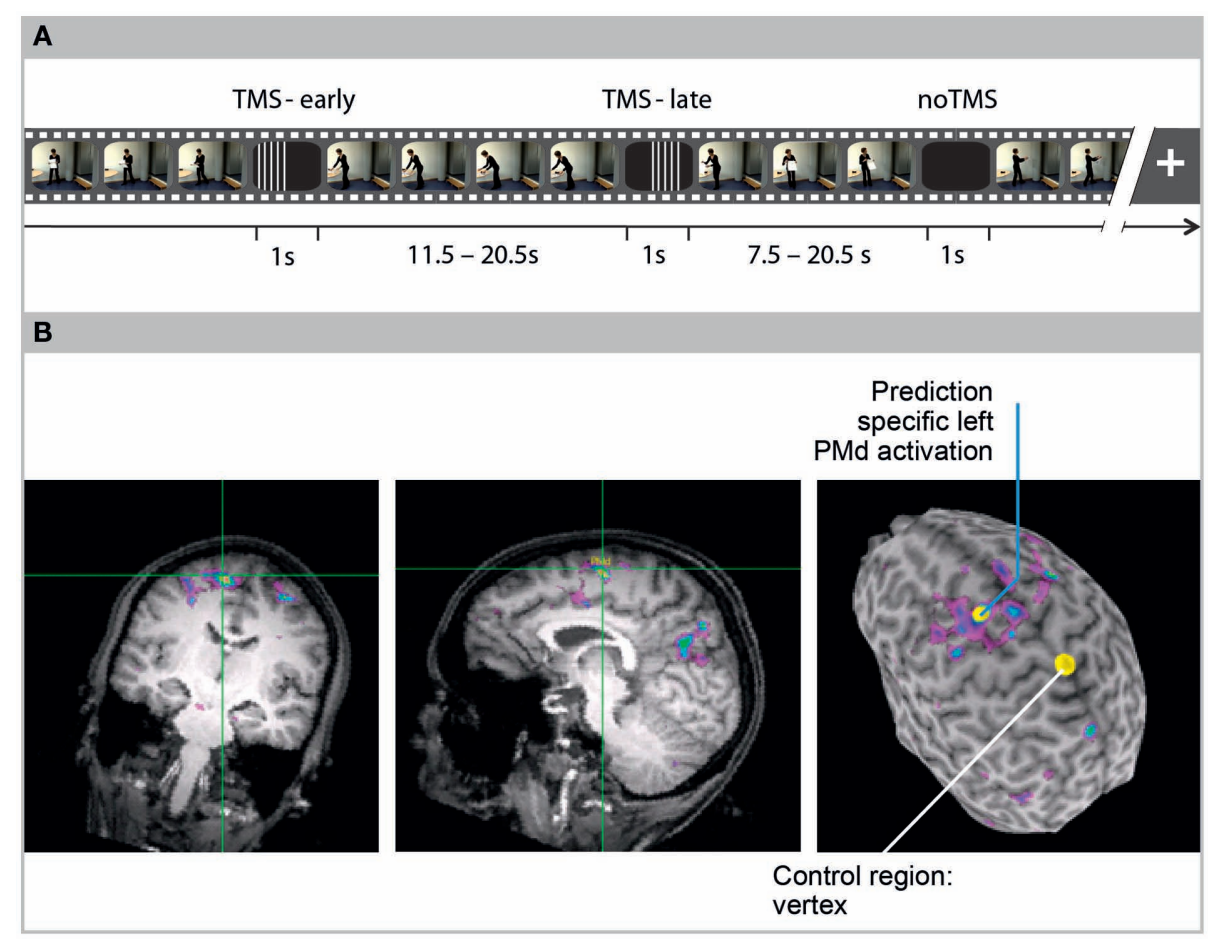

FIGURE 1 | (A) Design. Video clips showing naturalistic everyday actions were repeatedly occluded. Occlusions lasted for $1 \mathrm{~s}$. The actions continued immediately after the occlusions with either coherent or incoherent timing. In time-coherent trials, the action continuation corresponded to the occluder duration (i.e., the action continued $1 \mathrm{~s}$ after the last frame before occlusion). In time-incoherent trials, the action either continued at a frame that was too soon (coherent $-500 \mathrm{~ms}$ ) or belated in the action sequence

(coherent $+2000 \mathrm{~ms}$ ). Participants decided whether the action continued with coherent or incoherent timing, by pressing one of two response buttons with their left index finger. Repetitive TMS was applied only during occlusions in $50 \%$ of the trials. TMS application either started at occluder-onset (TMS-early) or $300 \mathrm{~ms}$ after occluder-onset (TMS-late). In 50\% of the trials (noTMS), performance under unstimulated conditions was assessed. Pulse trains included five single pulses at a frequency of $10 \mathrm{~Hz}$ and lasted for $400 \mathrm{~ms}$. The interval between occlusions varied randomly between 7.5 and 20.5 s. (B) $\mathrm{fMRl}$-guided coil navigation. A functional brain scan of one exemplary participant is overlaid on the participant's anatomical scan (coronal slice, sagittal slice, and 3D-rendered surface). In the 3D brain, yellow dots indicate the sites of TMS application, left dorsal premotor cortex (PMd) as the region of interest, and Vertex (VX) as the control region. For left PMd stimulation, the TMS coil was navigated to the local maximum obtained in an $\mathrm{fMRI}$ contrast between an action prediction task and an action memory task. too soon and belated continuations was motivated by pilot data showing that these values resulted in reasonable performance rates (of around $80 \%$ correctly answered trials).

The participants discriminated between coherently timed action continuations and incoherent ones by pressing one of two response buttons as soon as possible after occlusion, using their left index and middle fingers, respectively. Based on pilot experiments, the time shifts were chosen such that the overall error rates did not exceed $30 \%$ of all trials.

\section{TMS}

The resting motor threshold was assessed in each participant by recording motor-evoked potentials (MEPs) from the right first dorsal interosseous (FDI) muscle after applying single pulse TMS over the left primary motor cortex (M1). To this end, surface electrodes $(\mathrm{Ag} / \mathrm{AgCl})$ were placed over the muscle belly (i.e., active electrode) and over the corresponding tendon (i.e., reference) in a belly-tendon montage. The EMG was amplified at a gain of 1000, band-pass filtered $(20-2000 \mathrm{~Hz})$, and digitized at a sampling rate of $5 \mathrm{kHz}$. The resting motor threshold was defined as the lowest stimulation intensity to evoke MEPs of at least $50 \mu \mathrm{V}$ peak-to-peak in five out of 10 single pulse stimulations (Rossini et al., 1994). Stimulation was delivered using a figure-of-eight stimulation coil (70 mm, Magstim polyurethane coated) over the left M1, adjusting its position to optimize MEP amplitudes in response to single pulses. These were delivered with an inter-pulse delay of at least 5 s using a Magstim Rapid2 stimulator (The Magstim Company, Carmarthenshire, Wales, UK). Recording of EMG activity was continued throughout all experimental sessions.

While participants were performing the action prediction task, online rTMS was applied by means of a Magstim Air Film Coil (figure-of-eight shaped, $70 \mathrm{~mm}$ ) mounted on an articulated coil stand. A Magstim Rapid2 stimulator producing a maximum output of $3.5 \mathrm{~T}$ at the coil surface (output type: biphasic; pulse width: $400 \mu \mathrm{s}$ ) was used for stimulation at a frequency of $10 \mathrm{~Hz}$. Each train of pulses consisted of five single pulses with an interstimulus interval of $100 \mathrm{~ms}$, thus lasting for $400 \mathrm{~ms}$. Stimulation intensity was $110 \%$ of the individual resting motor threshold, ranging from 44 to $77 \%$ (mean $=62.1 \pm 10.1 \%$ ) of the maximum stimulator output. Intervals between pulse trains lasted for at least $12 \mathrm{~s}$ (respecting safety recommendations for the minimum delay between two rTMS trains; Rossi et al., 2009). In 
half of the rTMS trials, stimulation was delivered at the onset of an occluder (TMS-early condition), while in the other half, it was delivered $300 \mathrm{~ms}$ after the onset of an occluder (TMS-late condition).

For left PMd stimulation, the coil was positioned at the fMRI activation maximum that was found in each individual around the crossing of precentral sulcus with the superior frontal sulcus (SFS). The mean PMd coordinates, SD and ranges were $x=-22.6 \pm 6.7$ ( -14 to -39$), y=1.4 \pm 6.2$ ( -8 to 13$)$, and $z=53.8 \pm 2.6$ (5159; Talairach and Tournoux, 1988). The coil was oriented with an angle of $45^{\circ}$ to the sagittal axis. In order to control for unspecific effects of the rTMS treatment (such as tactile sensations and sound), the vertex area (VX) was chosen as a control site receiving rTMS stimulation with similar parameters as the PMd. For vertex stimulation, the coil was positioned over the sagittal midline, at the level of the postcentral gyri (Figure 1B). The mean VX coordinates, $\mathrm{SD}$, and ranges were $x=0.2 \pm 0.8$ ( -2 to 1 ), $y=-34.7 \pm 3.8$ $(-29$ to -42$)$, and $z=58.2 \pm 4.8$ (51-66).

The positioning of the rTMS coil on the regions of interest (ROIs) was navigated using fMRI of the individual participants in a frameless stereotaxic system including a Polaris IR tracker camera (Northern Digital, Waterloo, ON, Canada) that measured the position of anatomical landmarks on the participants' heads. These landmarks were co-registered with each participant's unpeeled anatomical MRI scan using Brainsight software (Rogue, Montreal, QC, Canada). The TMS coil was equipped with tracking points so that its position relative to the brain could be tracked online.

\section{MRI}

In order to obtain contrast images for coil navigation, fMRI was acquired in a separate session comprising two tasks (i.e., a prediction task and a memory task), both involving sequences of everyday actions that were performed by the same actress as those in the present study. The tasks were from an earlier study that found increased left PMd activation during action prediction (Stadler et al., 2011). More specifically, an action prediction task (similar to the one used in the present experiment) was employed in random alternation with a memory task in which the participants were required to memorize the last visible frame prior to the occlusion (for a more detailed description, see Stadler et al., 2011). Functional brain scans were acquired on a 3 T Bruker Medspec 30/100 system using a single shot gradient EPI sequence $(\mathrm{TE}=30 \mathrm{~ms}$, flip angle $90^{\circ}, \mathrm{TR}=2000 \mathrm{~ms}$, acquisition bandwidth $100 \mathrm{kHz}$ ). Twenty-two axial slices oriented parallel to the bicommissural plane were acquired (pixel matrix $=64 \times 64$, FOV $=19.2 \mathrm{~cm}$, inplane resolution $=3 \times 3 \mathrm{~mm}$, slice thickness $=4 \mathrm{~mm}$, interslice gap $=1 \mathrm{~mm}$ ). Data pre-processing and statistical analysis based on the general linear model were carried out with the software package LIPSIA (Lohmann et al., 2001). Contrast images were generated between target events (onsets of occlusions) of the two conditions Prediction and Memory. Functional MRI maps showing the contrast Prediction $>$ Memory in each individual were used for TMS coil navigation and were therefore overlaid on a high-resolution 3D reference dataset (EPI-T1). The brain was manually extracted and visualized for 3D surface rendering.

\section{PROCEDURE}

Half of the participants were in a previous fMRI experiment (Stadler et al., 2011) and contrast images used for localizing the ROIs during rTMS were already available for them. For the remaining six participants, an fMRI scan was acquired prior to the TMS experiment. The TMS experiment was conducted in two sessions. Within each session, only one ROI (left PMd or VX) was stimulated and the session order was counterbalanced over participants. Due to regulations of the local ethics commission, the participants in this study were not allowed to take part in a second brain imaging experiment within 1 week time. Consequently, the experimental sessions (fMRI, TMS 1, and TMS 2) were separated by 1 week. One day before the first TMS session, a 60 min practice session was held in which participants watched each video clip without occlusions, and then received feedback while practising the prediction task (36 trials, 17 with coherent timing) on two video clips (not used in the actual experiment). Afterward, they performed 40 trials (20 with coherent timing) that were similar to those of the TMS sessions (i.e., including the same videos and no feedback). The results of the 20 coherent trials were used to assess individual baseline performance.

The first TMS session lasted for about $90 \mathrm{~min}$ and started with the assessment of the resting motor threshold. The experimental part in which the prediction task was performed and rTMS was delivered either over the PMd or the VX was presented in three runs of 10 min duration that were separated by two short breaks.

In the second TMS session, the same stimulation parameters were used so it was not necessary to determine the motor threshold, resulting in a shorter overall duration of $45 \mathrm{~min}$.

In each session and per stimulated area (PMd and VX), a total number of 120 trials was presented, 60 of which were coherent, 30 too soon, and 30 belated. In both TMS sessions, half of the trials were without rTMS stimulation, which allowed us to rule out potential effects of anticipating rTMS application. Repetitive TMS trials were divided in 50\% TMS-early (i.e., rTMS at the onset of an occluder) and 50\% TMS-late trails (i.e., rTMS $300 \mathrm{~ms}$ after the onset of occlusion).

The software Presentation (Neurobehavioral SystemsTM, Albany, CA, USA) was used to control the presentation of visual stimuli, response registration, EMG acquisition, and for triggering TMS stimulation.

\section{ANALYSIS}

Effects of rTMS on error rates and RT were analyzed only for trials with coherent timing because performance rates were close to chance level in incoherent trials (mean $=60 \% \pm 14$ correct answers, $30-77 \%$ ), in contrast to $83 \% \pm 11$ (from 60 to $99 \%$ ) in coherent trials. The error rates and RTs in noTMS trials were used as measures of baseline performance; the performance in an initial practice session served as an additional baseline.

Error rates represent coherently timed continuations that were incorrectly judged as incoherent (i.e., missed). Two two-way repeated-measures ANOVAs were used to analyze the effects of rTMS-site (PMd vs. VX) and rTMS onset (TMS-early vs. TMS-late) on the error rates and the mean RTs as dependent variables, respectively. Additional two-way repeated-measures ANOVAs were used to assess effects of rTMS in contrast to noTMS 
trials, including the factors rTMS-site (PMd vs. VX) and interference (TMS, collapsed over TMS-early and TMS-late, vs. noTMS trials). Paired-samples $t$-tests (two-tailed) were used for comparisons between the factor levels. The influences of covariates were assessed by means of correlation analysis (Pearson, two-tailed) and by means of independent samples $t$-tests.

To control whether rTMS pulses elicited MEPs, EMG activity was analyzed within epochs from $100 \mathrm{~ms}$ before until $600 \mathrm{~ms}$ after the last rTMS pulse. The activity recorded before the first pulse was used as a baseline and this was subtracted from the activity obtained after pulse onset. For each participant, resulting amplitude values were then averaged over all rTMS trials within one session.

The software SPSS (IBM, SPSS statistics, Version 17) was used for statistical analyses.

\section{RESULTS}

\section{EFFECTS OF rTMS ON ERROR RATES}

The mean error rates are shown in Table $\mathbf{1}$ and are plotted in Figure 2 for the rTMS-sites (PMd and VX) and the rTMS onsets (TMS-early and TMS-late), respectively. In addition, error rates from noTMS trials (presented separately for the PMd and VX sessions) and from the pre-TMS practice session are shown as measures of baseline performance.

Error rates were highest when the PMd was stimulated in the TMS-early condition. However, no interaction effects were found between rTMS-site and rTMS onset $(P=0.19)$, which was possibly due to low statistical power. The TMS-induced increase in error rates was statistically significant when it was compared to the TMS-early condition in the VX $(t 10=2.79, P=0.019)$.

An additional two-way ANOVA which was carried out to compare rTMS trials with noTMS trials, including the factors rTMSsite (PMd, VX) and interference (TMS, noTMS), also did not show any significant effects.

In a next step, the influence of several independent variables on the error rates during TMS-early and TMS-late in PMd and VX was studied by means of correlation analysis (Pearson correlation). These variables were (i) the localization of the rTMS focus in the anterior-to-posterior dimension ( $y$-coordinate) and in the medial-to-lateral dimension ( $x$-coordinate), (ii) the strength of the fMRI contrast at the stimulated site, and (iii) the resting motor threshold, which determined stimulation intensity in each individual. The only significant correlation found was a negative correlation between TMS-early in VX and the resting motor threshold $(r 11=-0.62 ; P=0.043)$. Thus, the higher the motor threshold (i.e., the stimulation intensity), the lower the error rates when the VX was stimulated during occlusion.

The effect of session order (i.e., whether the PMd was targeted in the first or the second TMS session) was assessed by means of an independent samples $t$-test comparing error rates during PMd stimulation in the first session to error rates during PMd stimulation in the second session. No significant difference was found.

\section{EFFECTS OF rTMS ON RTs}

The mean RTs are shown in Table 2 and plotted in Figure 3 for the rTMS-sites (PMd and VX) and the rTMS onset (TMS-early and TMS-late). In addition, RTs for noTMS trials (presented separately for the PMd and VX sessions) and RTs for the pre-TMS practice session are shown. No RT differences were found between rTMS-sites and rTMS onsets. However, mean RTs were longer in the baseline condition and during noTMS trials as compared to TMS trials in both the PMd and the VX sessions. This difference was statistically confirmed in a two-way ANOVA with the factors rTMS-site (PMd, VX) and interference (TMS trials, noTMS trials), revealing a significant main effect of interference $(F 1,10=6.21$, $P=0.032$ ). Thus, the RTs were generally reduced in TMS trials, irrespective of the site stimulated and TMS onset.

In addition, the influence of several independent variables on RT was assessed by means of correlation analysis (Pearson correlation), with the variables (i) localization of rTMS focus in the anterior-to-posterior dimension ( $y$-coordinate) and in the medial-to-lateral dimension ( $x$-coordinate), (ii) strength of the fMRI contrast at the stimulated site, and (iii) resting motor threshold. None of these correlated significantly with the RTs measured in the different conditions.

Further, no significant differences were found when analyzing the influence of the session order by means of an independent samples $t$-test comparing RT when PMd was stimulated in the first session to RT when PMd was stimulated in the second session.

\section{EMG ACTIVITY}

Electromyographic activity in response to rTMS trains was recorded from the FDI muscle of the right hand and was compared between the rTMS-sites. Repetitive TMS over the left PMd elicited significantly higher EMG activity than VX stimulation $(t 10=2.59, P=0.027)$. In contrast to VX stimulation, left PMd stimulation occasionally evoked MEPs in some participants (left

Table 1 | Mean error rates (\%) and SE forTMS (TMS-early, TMS-late), and noTMS trials for the two TMS sessions (PMd and VX) and for the practice session.

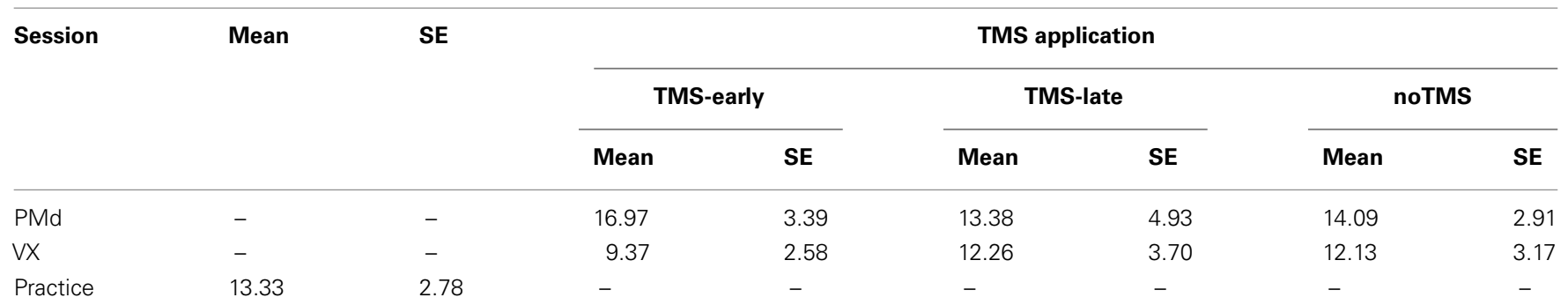


PMd: mean EMG amplitude $=380 \mu \mathrm{V}, \mathrm{SD}=441$; VX: mean EMG amplitude $=37 \mu \mathrm{V}, \mathrm{SD}=22$ ). However, no evidence was found of an influence of EMG activity on performance. EMG amplitudes neither correlated significantly with error rates (PMd stimulation: $r 11=0.25, P=0.46$; VX stimulation: $r 11=0.50, P=0.12$ ) nor with RT (PMd stimulation: $r 11=-0.25, P=0.46$; VX stimulation: $r 11=0.01, P=0.99$; TMS trials were collapsed over early and late stimulation).

\section{DISCUSSION}

We used rTMS to study the functional significance of the PMd for action prediction. To this end, an "occluder-paradigm" was set up, using video clips of everyday actions that were repeatedly occluded for $1 \mathrm{~s}$. Occlusions covered object-directed arm and full-body actions performed by a right-handed actress. The participants' task was to judge whether or not the action continued with coherent timing after an occlusion. The time course of the actions was occasionally manipulated during occlusion, resulting in action continuations that were too soon or too advanced relative to the undisrupted action. In two conditions, rTMS was delivered to the left PMd either at the onset of occlusion (TMS-early) or $300 \mathrm{~ms}$ after the onset of occlusion (TMS-late). In a control session, the same procedure was applied while stimulating the VX.

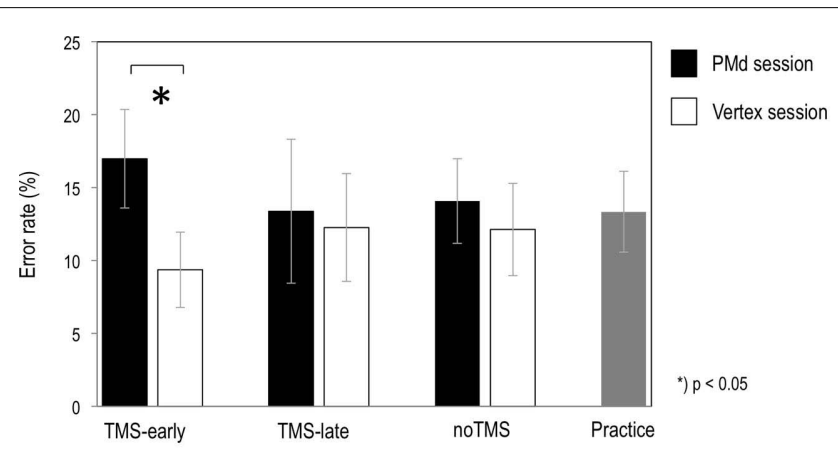

FIGURE 2 | Graphs show the mean error rates for TMS-early and TMS-late in the left PMd and in the VX. Error rates from noTMS trials (i.e., trials without TMS that were randomly intermixed between TMS trials in each session) and from the practice session were taken as measures of baseline performance. Errors were those cases when a time-coherent action continuation was misjudged as being time-incoherent. Bars represent SE.
Even though the present results lack the fortification of strong statistical confirmation (only one pairwise comparison in a $2 \times 2$ factorial design was significant), they might inform future research and highlight interesting functional interpretations of the PMd from earlier literature which is discussed below. In the TMS-early condition, rTMS over the PMd led to an increased number of prediction errors when compared to VX stimulation. More precisely, participants had an increased tendency to falsely judge time-coherent action continuations as incoherent. Interestingly, rTMS had this effect when it was applied at the onset of occlusions, but not when it was applied $300 \mathrm{~ms}$ after the onset of occlusions. In agreement with our hypothesis, this result suggests that the PMd is indeed functionally relevant for generating predictions about the future course of observed action sequences. However, before discussing the results in detail we have to point out limitations in statistical confirmation. In particular, a significant effect of TMS was only found in a pairwise comparison between PMd and VX stimulation in the TMS-early condition. Error rates increased specifically after early rTMS over the PMd and were lowest after TMS-early over the VX. Nevertheless, the overall interaction between rTMS-site and rTMS onset did not reach significance. The lack of a statistically significant interaction is probably due to low statistical power which leads to an increased possibility of a type II error. Although this raises reservations, it should be considered that the pattern of results draws a consistent picture which is in good correspondence with the relevant literature.

Irrespective of its onset and the cortical site stimulation was delivered to, RTs were generally shorter in rTMS trials than in noTMS trials. This finding stands in contrast to the absence of RT shortening during visuomotor learning which was found specifically after TMS interference in the right PMd (Praeg et al., 2005). In the present study, the RT shortening was not specific to the stimulated site and occurred in all conditions. Different effects of TMS to the left PMd compared to the right PMd could point to different functional roles that these areas play during sensorimotor integration. In accordance with the present findings, unspecific RT shortening was reported for simple RT (Terao et al., 1997), go/nogo RT (Sawaki et al., 1999) and sequential responses (Gregori et al., 2005). In these studies, non-specific RT shortening occurred independently of the rTMS application site and was observed after both subthreshold and suprathreshold stimulation. The non-specific RT effects were hence explained by intersensory facilitation (e.g., Nickerson, 1973), which refers to additive facilitating effects of

Table 2 | Mean reaction times and SE for TMS (TMS-early, TMS-late) and noTMS trials, for the two TMS sessions (PMd and VX) and for the practice session.

\begin{tabular}{|c|c|c|c|c|c|c|c|c|}
\hline \multirow[t]{2}{*}{ Session } & \multirow[t]{2}{*}{ Mean } & \multirow[t]{2}{*}{ SE } & \multicolumn{6}{|c|}{ TMS application } \\
\hline & & & \multicolumn{2}{|c|}{ TMS-early } & \multicolumn{2}{|c|}{ TMS-late } & \multicolumn{2}{|c|}{ notMS } \\
\hline PMd & - & - & 1021.71 & 36.68 & 998.92 & 42.48 & 1045.19 & 44.41 \\
\hline VX & - & - & 1019.67 & 41.56 & 1011.47 & 53.37 & 1054.15 & 46.77 \\
\hline
\end{tabular}




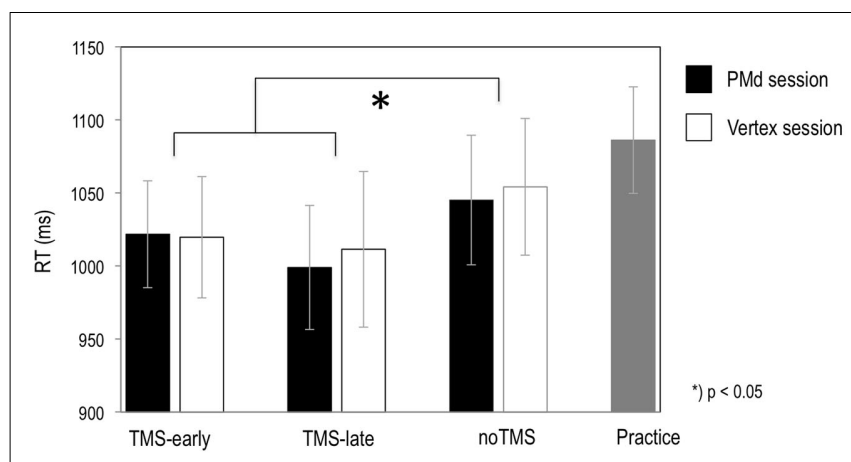

FIGURE 3 | Graphs show the mean RTs for TMS-early and TMS-late in the left PMd and in the VX for trials with coherently timed action continuations after occlusion. RTs from noTMS trials (i.e., trials without TMS that were randomly intermixed between TMS trials in each session) and from the practice session were considered as measures of baseline performance. Bars represent SE.

multimodal sensory stimulation that is applied simultaneously. During rTMS, the discharging coil produces tactile sensations at the scalp which are accompanied by acoustic clicks. In the present study, not only the mean RT was shorter in rTMS trials compared to noTMS trials, but also the error rates in the TMS-early condition of the VX session decreased with stronger stimulation intensity. This was expressed in a significant correlation between error rates and resting motor threshold. In fact, the tactile sensation and the clicking sound increase with stimulation intensity. This interpretation suggests that while the participants might have been generally more efficient in TMS trials, the interference in the PMd at early times during occlusion could have inhibited particularly those functions that were required in the action prediction task.

Conversely, TMS over the VX could have induced modulation in mesial superior parietal areas, such as the precuneus (PCu; Cavanna and Trimble, 2006) or more laterally in the superior parietal lobe (SPL; Rushworth et al., 2003; Busan et al., 2009). Both areas are involved in visuo-spatial processing during actionrelated tasks. The increase in accuracy with the increasing intensity of VX stimulation might reflect either the facilitation of task relevant processing (Busan et al., 2009) or the inhibition of redundant functions (Oshio et al., 2010).

As indicated in the EMG activity in the right FDI that was significantly increased during PMd stimulation, TMS over the left PMd modulated the activation in the ipsilateral M1, occasionally eliciting MEPs. Such propagation of activity to M1 is not surprising, given its dense connectivity to premotor areas (e.g., Wise et al., 1997; Picard and Strick, 2001). It has been shown before that TMS to the premotor cortex can induce excitability changes in the ipsilateral primary motor cortex (Civardi et al., 2001; Rizzo et al., 2004). Note that EMG was measured in the FDI muscle of the right hand, contralateral to the stimulated (left) hemisphere, and responses were given with the ipsilateral (left) hand. Also, transcallosal propagation to the contralateral motor cortex was observed after PMd stimulation (Koch and Rothwell, 2009). Here, it can not be excluded that PMd stimulation influenced the participants' response behavior in this way, although we did not find any indication (such as RT differences between stimulation sites).

\section{CONTRIBUTION OF THE LEFT PMd TO ACTION PREDICTION}

The present results provide first insights to the way in which the PMd might contribute to action prediction. The study was designed to complement the findings of an earlier fMRI study showing that BOLD activation in this area was strongest during an action prediction task, as compared to control conditions which employed the same stimuli but different task instructions (e.g., memory task; Stadler et al., 2011).

Previous studies targeted the PMv to disrupt the sensorimotor transformation of perceived action information. For instance, TMS over the PMv affected weight judgments of a lifted box (Pobric and Hamilton, 2006) and disrupted the discrimination of upright static pictures showing dancing postures (Urgesi et al., $2007 \mathrm{~b}$ ) and the visual discrimination of actions presented dynamically and in static images (Avenanti et al., 2007; Urgesi et al., 2007a; Candidi et al., 2008). However, in contrast to the PMv, the PMd seems to be concerned with movement requiring a higher level of integration such as guiding the arm toward an object in space. These differential specializations are well documented for motor control (Davare et al., 2006; Hoshi and Tanji, 2007) and were also found during action perception (Buccino et al., 2001; Sakreida et al., 2005). Corresponding actions were occluded in the present study. Thus, the performance increment after PMd stimulation might reflect prediction that is based on simulating the occluded motor actions (Graf et al., 2007). Applying the action simulation account to the present study, suggests that motor programs involved in controlling right arm movements were activated during occlusion and produced predictions of the sensory consequences of motor actions (Grush, 2004; Wilson and Knoblich, 2005). When the action continued after occlusion, the prediction was matched with the actual action continuation.

TMS studies that disrupted PMd function during motor control found effects on the timing of arm movements (Davare et al. 2006) and on the anticipatory scaling of forces when objects with different weights were lifted (Chouinard et al., 2005). Since object weight was indicated by visual symbols in the latter study, impaired force scaling could be attributed to the ineffective use of parameters for arm movements that were provided by the visual cues (e.g., Kalaska et al., 1997). In other studies, TMS was applied over the left PMd during choice reaction time tasks and led to prolonged RTs of both ipsilateral and contralateral hand responses (Schluter et al., 1998; Johansen-Berg et al., 2002). Thus, some of these results provide evidence of effector-unspecific motorcognitive functions of the left PMd which has been corroborated by imaging studies (e.g., Grafton et al., 2002; Sakai et al., 2002; Ohbayashi et al., 2003; Haaland et al., 2004). These imaging studies involve the generation of more or less complex finger-tapping sequences that are usually instructed by a visual stimulus sequence. Some authors associated the PMd with action selection according to learned (arbitrary) associations between (usually visual) stimuli and motor responses (see Rushworth et al., 2003 for a review). Other authors relate PMd activation to the internal organization 
of action sequences, especially their ordinal (or temporal) structuring (e.g., Ohbayashi et al., 2003; Haaland et al., 2004). The PMd was further found to contribute to purely cognitive tasks that require visuo-spatial transformations, such as mental rotation (Lamm et al., 2001; Abe et al., 2007) or serial prediction based on spatial stimulus properties (Schubotz and von Cramon, 2001; Schubotz et al., 2003) and TMS over the PMd can affect the accuracy in mental rotation tasks (Oshio et al., 2010). These functional requirements, selection, structuring, and spatial transformation, seem to be compatible with the particular demands of the present prediction task. Here, occluded actions were internally substituted, requiring observers to mentally generate an action sequence under internal (i.e., memory-based) guidance (Springer and Prinz, 2010). The parameters determining the selection of the appropriate action components (Cisek, 2007) were given in the particular video scene perceived prior to occlusion.

To conclude, the PMd, an area that is involved in the control of arm and full-body actions, might be needed when actions of this kind have to be predicted during observation. The correspondence between action execution and action observation might either point to the direct activation of motor programs to simulate the observed/occluded actions or, alternatively, to the access of effector-unspecific motor-cognitive functions of this particular area.

\section{TIMING OF THE LEFT PMd CONTRIBUTION TO ACTION PREDICTION}

The time point of the rTMS application was crucial. Repetitive TMS over the left PMd only affected action prediction when it was delivered at the onset of the occlusion, taken to reflect an initial phase of internal action prediction. First, this observation relates to studies that showed RT slowing following PMd stimulation at short latencies after the presentation of a visual cue (Schluter et al., 1998, 1999; Johansen-Berg et al., 2002). Further, the PMd might be involved particularly in the initiation of prediction, which is in line with an idea recently put forward by Prinz and Rapinett (2008). The authors found that real-time simulation of occluded actions (Graf et al., 2007) can lag behind real-time by a constant latency (of about 20-120 ms; Sparenberg et al., 2012). The concept of real-time simulation claims that observed actions are mentally simulated in real-time in order to achieve internal predictions (Graf et al., 2007). Prinz and Rapinett (2008) specified this concept by proposing that the positive time error reflects a restart of the internal simulation. Accordingly, real-time simulation runs continuously during ongoing observation and is restarted when the observed action is occluded. Drawing on the restart hypothesis, one may take the present results to suggest that PMd is involved in restarting (i.e., for initiating the simulation of an action sequence in a purely internally guided mode).

A related issue was studied by Kennerley et al. (2004) during motor control. They aimed to investigate the roles of the preSMA and the left PMd during the initiation of finger-tapping sequences and therefore used rTMS. Their work was derived from evidence from RT analysis suggesting that movement sequences are at least partly programmed prior to the start of the first movement. This is, for instance, indicated by a relatively long RT for the first movement in a sequence, with an RT increase that is proportional to the total length of the sequence (e.g., Sternberg et al., 1978; Rosenbaum et al., 1983). Kennerley et al. (2004) found that disrupting pre-SMA functions, but not PMd functions, prolonged sequence initiation only during internal guidance (i.e., when the sequence was retrieved from memory and not when it was externally cued). The results obtained by Kennerley and colleagues appear to be inconsistent with the present results, since in their study, only rTMS over the pre-SMA but not left PMd had an effect on action initiation. However, the different experimental designs could account for this apparent discrepancy. Kennerley et al. (2004) employed a task that required the generation of sequential finger movements according to a sequence that was previously memorized. In contrast, in the present experiment, the predicted actions were determined by parameters given in a complex spatial setup that was shown in naturalistic video clips. Object-directed actions call on the participation of lateral premotor areas (PMd and PMv) due to their relevance in achieving sensorimotor transformations. While the pre-SMA is associated with internal guidance, lateral premotor-based action control relies on perceptual information to specify action parameters (Goldberg, 1985; Matelli and Luppino, 2001; Ogawa et al., 2006; Gowen and Miall, 2007). The PMd, in particular, is part of a network underlying the transformation of visuo-spatial information into motor codes (Wise et al., 1997; Matelli and Luppino, 2001; Pesaran et al., 2006; Tomassini et al., 2007). Thus, we assume that for predicting the time course of actions directed toward objects in space (as depicted in the video scenes), the PMd integrates action parameters that are extracted from the current scene just as it does during reach planning (Beurze et al., 2007; Hoshi and Tanji, 2007).

Disturbing the left PMd might result in an interference with the initial spatial and temporal programming of the action continuation according to action-relevant parameters. These actionrelevant parameters, such as posture, the orientation of the body, and the objects in the scene and spatial distances between them, can be provided to the PMd via projections from posterior parietal areas (e.g., Fagg and Arbib, 1998; Matelli and Luppino, 2001; Tomassini et al., 2007). Note that the constant occluder duration of $1 \mathrm{~s}$ may have facilitated such initial programming, since at the onset of occlusions, participants were aware of how long the predicted sequence would last. At the moment when TMS-late was applied, such integration with external parameters may have been terminated. TMS-late had no effect on prediction performance, suggesting that the PMd did not contribute significantly in the middle of occlusions, when ongoing action prediction was maintained. Presumably, this function may be achieved by other nodes of the action observation/imagery network, which could be an issue for future research.

Inspired by ideas of other authors (Wise et al., 1997; Grol et al., 2006), we speculate that the integrative functions of the PMd may be particularly appropriate for initiating large-scale prediction (i.e., prediction over temporal intervals in the range of seconds), which seems to be in accordance with its involvement in controlling actions that cover relatively wide spatial distances (in contrast to coordinated finger movements as represented in PMv) and in movement that requires the integration of a larger number of joints. 


\section{LIMITATIONS AND PERSPECTIVES}

The most obvious limitation of the present study is the comparably low statistical power of the data. This reservation should be kept in mind when considering the interpretations of PMd involvement in the initiation of action prediction provided here. We take this study to suggest a way in which functional interpretations of the PMd from literature might fit to the domain of action prediction. Further research is needed to strengthen this approach.

Finding TMS over the PMd to excite the FDI muscle of the contralateral hand raises the question to which extent modulations in other interconnected areas or in interregional network activity contributed to the observed behavioral effects (e.g., see Siebner et al., 2009). Moreover, after disrupting the left PMd by means of offline rTMS, compensatory activation can occur in the contralateral premotor cortex (O'Shea et al., 2007). The fact that TMS effects are not locally restricted imposes a certain reservation toward functional conclusions drawn from the application of focal TMS. This holds as long as the complex modulations in neural networks induced by TMS can not be entirely assessed.

Another issue concerns the selection of the control site which requires weighing of costs against benefits. The VX was chosen as a control region for several reasons. Primarily, we found no prediction specific fMRI activation in this area. This does not exclude that TMS over the VX interfered in functions that, although not specific to action prediction, were still task relevant. As outlined above, VX stimulation might have modulated functions represented in mesial or lateral superior parietal areas. However, the current data do not provide indications to specify possible effects. A second argument for choosing VX as a control site are weak sensory side effects of TMS that are comparable to those of PMd stimulation.

An alternative comparison is between $\mathrm{PMd}$ and another region of the action observation network (AON). Areas in this network are also active during mental imagery of actions and when occluded actions are predicted (Grèzes and Decety, 2001; Cross et al., 2011; Stadler et al., 2011). Further research could clarify

\section{REFERENCES}

Abe, M., Hanakawa, T., Takayama, Y., Kuroki, C., Ogawa, S., and Fukuyama, H. (2007). Functional coupling of human prefrontal and premotor areas during cognitive manipulation. J. Neurosci. 27, 3429-3438.

Avenanti, A., Bolognini, N., Maravita, A., and Aglioti, S. M. (2007). Somatic and motor components of action simulation. Curr. Biol. 17, 2129-2135.

Beurze, S. M., de Lange, F. P., Toni, I., and Medendorp, W. P. (2007). Integration of target and effector information in the human brain during reach planning. J. Neurophysiol. 97, 188-199.

Bode, S., Koeneke, S., and Jäncke, L. (2007). Different strategies do not moderate primary motor cortex

their role relative to that of the PMd during action prediction. For instance, the PMv that was shown to be activated during the occlusion of observed grasping actions in monkeys (Umiltà et al., 2001) might be relevant when different action types (e.g., object manipulation, instead of more spatially defined arm actions and translational actions) are predicted (Davare et al., 2006). Posterior regions in the AON such as the human pSTS which was involved during longer lasting (i.e., $3 \mathrm{~s}$ ) occlusion of intransitive actions (Saxe et al., 2004), might supply input to premotor areas during prediction. Compared to the PMd, this area could be relevant in different phases of the action prediction task.

\section{SUMMARY}

The present study highlights how the left PMd might contribute to predicting action in the absence of visual information. Particularly, the time point is indicated at which left PMd functions might be required. More precisely, we suggest that the left PMd might be involved in initiating predictions at the moment when an observed action becomes occluded but may hand over to other regions when ongoing prediction has to be maintained. This interpretation is consistent with the function that is ascribed to the PMd in motor control, which is the transformation of visuo-spatial or learned arbitrary information into motor codes. With reference to the action parameters that are achieved during such transformations, the PMd may contribute to the initial programming of the predicted action. Overall, this interpretation is in accordance with the notion that the prediction of observed dynamics employs similar structures to those required to overtly produce dynamics (Schubotz, 2007), which is in line with the common coding principle (Prinz, 1997) and with the simulation account (Jeannerod, 2001). It is also consistent with human mirror system research (e.g., Buccino et al., 2001; Fadiga et al., 2005).

\section{ACKNOWLEDGMENTS}

The authors would like to thank Jonas Petermann for lab assistance, Stephan Liebig for aid with the artwork, Rosie Wallis for proof reading, and Sarah Schräder for acting.

of ventral premotor cortex impairs visual perception of biomechanically possible but not impossible actions. Soc. Neurosci. 3, 388-400.

Cavanna, A. E., and Trimble, M. R. (2006). The precuneus: a review of its functional anatomy and behavioural correlates. Brain 129, 564-583.

Chouinard, P. A., Leonard, G., and Paus, T. (2005). Role of the primary motor and dorsal premotor cortices in the anticipation of forces during object lifting. J. Neurosci. 25, 2277-2284.

Cisek, P. (2007). Cortical mechanisms of action selection: the affordance competition hypothesis. Philos. Trans. R. Soc. Lond. B Biol. Sci. 362, 1585-1599.

Cisek, P., and Kalaska, J. F. (2004). Neural correlates of mental rehearsal in dorsal premotor cortex. Nature 431, 993-996.

Civardi, C., Cantello, R., Asselman, P., and Rothwell, J. C. (2001). Transcranial magnetic stimulation can be used to test connections to primary motor areas from frontal and medial cortex in humans. Neuroimage 14, 1444-1453.

Cross, E. S., Stadler, W., Parkinson, J., Schütz-Bosbach, S., and Prinz, W. (2011). The influence of visual training on predicting complex action sequences. Hum. Brain Mapp. doi: 10.1002/hbm. 21450

Davare, M., Andres, M., Cosnard, G., Thonnard, J. L., and Olivier, E. (2006). Dissociating the role of ventral and dorsal premotor cortex in precision grasping. J. Neurosci. 26, 2260-2268. 
Eisenegger, C., Herwig, U., and Jäncke, L. (2007). The involvement of primary motor cortex in mental rotation revealed by transcranial magnetic stimulation. Eur. J. Neurosci. 25, 1240-1244.

Fadiga, L., Craighero, L., and Olivier, E. (2005). Human motor cortex excitability during the perception of others' action. Curr. Opin. Neurobiol. $15,213-218$.

Fagg, A., and Arbib, M. (1998). Modeling parietal-premotor interactions in primate control of grasping. Neural. Netw. 11, 1277-1303.

Filimon, F., Nelson, J. D., Hagler, D. J., and Sereno, M. I. (2007). Human cortical representations for reaching: mirror neurons for execution, observation, and imagery. Neuroimage 37, 1315-1328.

Goldberg, G. (1985). Supplementary motor area structure and function: review and hypotheses. Behav. Brain Sci. 8, 567-616.

Gowen, E., and Miall, R. C. (2007). Differentiation between external and internal cuing: an fMRI study comparing tracing with drawing. $\mathrm{Neu}$ roimage 36, 396-410.

Graf, M., Reitzner, B., Corves, C., Casile, A., Giese, M., and Prinz, W. (2007). Predicting point-light actions in real-time. Neuroimage 36(Suppl. 2), T22-T32.

Grafton, S. T., Hazeltine, E., and Ivry, R. B. (2002). Motor sequence learning with the nondominant left hand. A PET functional imaging study. Exp. Brain Res. 146, 369-378.

Gregori, B., Currà, A., Dinapoli, L., Bologna, M., Accornero, N., and Berardelli, A. (2005). The timing and intensity of transcranial magnetic stimulation, and the scalp site stimulated, as variables influencing motor sequence performance in healthy subjects. Exp. Brain Res. 166, 43-55.

Grèzes, J., and Decety, J. (2001). Functional anatomy of execution, mental simulation, observation, and verb generation of actions: a metaanalysis. Hum. Brain Mapp. 12, 1-19.

Grol, M. J., de Lange, F. P., Verstraten, F. A., Passingham, R. E., and Toni, I. (2006). Cerebral changes during performance of overlearned arbitrary visuomotor associations. J. Neurosci. 26, 117-125.

Grush, R. (2004). The emulation theory of representation: motor control, imagery, and perception. Behav. Brain Sci. 27, 377-442.

Haaland, K. Y., Elsinger, C. L., Mayer, A. R., Durgerian, S., and Rao, S. M. (2004). Motor sequence complexity and performing hand produce differential patterns of hemispheric lateralization. J. Cogn. Neurosci. 16, 621-636.

Hoshi, E., and Tanji, J. (2000). Integration of target and body-part information in the premotor cortex when planning action. Nature 408, 466-470.

Hoshi, E., and Tanji, J. (2007). Distinctions between dorsal and ventral premotor areas: anatomical connectivity and functional properties. Curr. Opin. Neurobiol. 17, 234-242.

Jeannerod, M. (2001). Neural simulation of action: a unifying mechanism for motor cognition. Neuroimage 14, S103-S109.

Johansen-Berg, H., Rushworth, M. F., Bogdanovic, M. D., Kischka, U., Wimalaratna, S., and Matthews, P. M. (2002). The role of ipsilateral premotor cortex in hand movement after stroke. Proc. Natl. Acad. Sci. U.S.A. 99, 14518-14523.

Kalaska, J. F., Scott, S. H., Cisek, P., and Sergio, L. E. (1997). Cortical control of reaching movements. Curr. Opin. Neurobiol. 7, 849-859.

Kennerley, S. W., Sakai, K., and Rushworth, M. F. (2004). Organization of action sequences and the role of the pre-SMA. J. Neurophysiol. 91, 978-993.

Koch, G., and Rothwell, J. C. (2009). TMS investigations into the taskdependent functional interplay between human posterior parietal and motor cortex. Behav. Brain Res. 202, 147-152.

Lamm, C., Windischberger, C., Leodolter, U., Moser, E., and Bauer, H. (2001). Evidence for premotor cortex activity during dynamic visuospatial imagery from singletrial functional magnetic resonance imaging and event-related slow cortical potentials. Neuroimage 14, 268-283.

Lohmann, G., Muller, K., Bosch, V., Mentzel, H., Hessler, S., Chen, L., Zysset, S., and von Cramon, D. Y. (2001). LIPSIA - a new software system for the evaluation of functional magnetic resonance images of the human brain. Comput. Med. Imaging Graph. 25, 449-457.

Matelli, M., and Luppino, G. (2001). Parietofrontal circuits for action and space perception in the macaque monkey. Neuroimage 14, S27-S32.

Nickerson, R. S. (1973). Intersensory facilitation of reaction time: energy summation or preparation enhancement? Psychol. Rev. 80, 489-509.

Ogawa, K., Inui, T., and Sugio, T. (2006). Separating brain regions involved in internally guided and visual feedback control of moving effectors: an event-related fMRI study. Neuroim age 32, 1760-1770.

Ohbayashi, M., Ohki, K., and Miyashita, Y. (2003). Conversion of working memory to motor sequence in the monkey premotor cortex. Science 301, 233-236.

Oldfield, R. (1971). Assessment and analysis of handedness - Edinburgh inventory. Neuropsychologia 9, 97-113.

O'Shea, J., Johansen-Berg, H., Trief, D., Gobel, S., and Rushworth, M. F. (2007). Functionally specific reorganization in human premotor cortex Neuron 54, 479-490.

Oshio, R., Tanaka, S., Sadato, N., Sokabe, M., Hanakawa, T., and Honda, M (2010). Differential effect of doublepulse TMS applied to dorsal premotor cortex and precuneus during internal operation of visuospatial information. Neuroimage 49, 1108-1115.

Pesaran, B., Nelson, M., and Andersen, R. (2006). Dorsal premotor neurons encode the relative position of the hand, eye, and goal during reach planning. Neuron 51, 125-134.

Picard, N., and Strick, P. L. (2001). Imaging the premotor areas. Curr. Opin. Neurobiol. 11, 663-672.

Pobric, G., and Hamilton, A. (2006). Action understanding requires the left inferior frontal cortex. Curr. Biol. 16, 524-529.

Praeg, E., Herwig, U., Lutz, K., and Jancke, L. (2005). The role of the right dorsal premotor cortex in visuomotor learning: a transcranial magnetic stimulation study. Neuroreport 16, 1715-1718.

Prinz, W. (1997). Perception and action planning. Eur. J. Cogn. Psychol. 9, 129-154.

Prinz, W., and Rapinett, G. (2008). "Filling the gap: dynamic representation of occluded action," in Enacting Intersubjectivity: A Cognitive and Social Perspective on the Study of Interactions, eds F. Morganti, A. Carassa, and G. Riva (Amsterdam: IOS Press), 223-236.

Raos, V., Franchi, G., Gallese, V., and Fogassi, L. (2003). Somatotopic organization of the lateral part of area F2 (dorsal premotor cortex) of the macaque monkey. J. Neurophysiol. 89, 1503-1518.

Rizzo, V., Siebner, H. R., Modugno, N., Pesenti, A., Münchau, A., Gerschlager, W., Webb, R. M., and Rothwell, J. C. (2004). Shaping the excitability of human motor cortex with premotor rTMS. J. Physiol. (Lond.) 554, 483-495.
Rosenbaum, D. A., Kenny, S. B., and Derr, M. A. (1983). Hierarchical control of rapid movement sequences. $J$. Exp. Psychol. Hum. Percept. Perform. 9, 86-102.

Rossi, S., Hallett, M., Rossini, P. M. Pascual-Leone, A., and Safety of TMS Consensus Group. (2009). Safety, ethical considerations, and application guidelines for the use of transcranial magnetic stimulation in clinical practice and research. Clin. Neurophysiol. 120, 2008-2039.

Rossini, P. M., Barker, A. T., Berardelli, A., Caramia, M. D., Caruso, G., Cracco, R. Q., Dimitrijevic, M. R., Hallett, M., Katayama, Y., and Lücking, C. H. (1994). Non-invasive electrical and magnetic stimulation of the brain, spinal cord and roots: basic principles and procedures for routine clinical application. Report of an IFCN committee. Electroencephalogr. Clin. Neurophysiol. 91, 79-92.

Rushworth, M., Johansen-Berg, H., Gobel, S., and Devlin, J. (2003). The left parietal and premotor cortices: motor attention and selection. Neuroimage 20(Suppl. 1), S89-S100. Sakai, K., Ramnani, N., and Passingham, R. E. (2002). Learning of sequences of finger movements and timing: frontal lobe and action-oriented representation. J. Neurophysiol. 88, 2035-2046.

Sakreida, K., Schubotz, R. I., Wolfensteller, U., and von Cramon, D. Y (2005). Motion class dependency in observers' motor areas revealed by functional magnetic resonance imaging. J. Neurosci. 25, 1335-1342.

Sawaki, L., Okita, T., Fujiwara, M., and Mizuno, K. (1999). Specific and non-specific effects of transcranial magnetic stimulation on simple and go/no-go reaction time. Exp. Brain Res. 127, 402-408.

Saxe, R., Xiao, D. K., Kovacs, G., Perrett, D. I., and Kanwisher, N. (2004). A region of right posterior superior temporal sulcus responds to observed intentional actions. Neuropsychologia 42, 1435-1446.

Schluter, N. D., Rushworth, M. F., Mills, K. R., and Passingham, R. E. (1999). Signal-, set-, and movementrelated activity in the human premotor cortex. Neuropsychologia 37, 233-243.

Schluter, N. D., Rushworth, M. F., Passingham, R. E., and Mills, K. R. (1998). Temporary interference in human lateral premotor cortex suggests dominance for the selection of movements. A study using transcranial magnetic stimulation. Brain 121, 785-799. 
Schubotz, R. I. (2007). Prediction of external events with our motor system: towards a new framework. Trends. Cogn. Sci. (Regul. Ed.) 11, 211-218.

Schubotz, R. I., and von Cramon, D. Y. (2001). Functional organization of the lateral premotor cortex: fMRI reveals different regions activated by anticipation of object properties, location and speed. Brain Res. Cogn. Brain Res. 11, 97-112.

Schubotz, R. I., von Cramon, D. Y., and Lohmann, G. (2003). Auditory what, where, and when: a sensory somatotopy in lateral premotor cortex. Neuroimage 20, 173-185.

Schütz-Bosbach, S., and Prinz, W. (2007). Prospective coding in event representation. Cogn. Process. 8, 93-102.

Siebner, H. R., Hartwigsen, G., Kassuba, T., and Rothwell, J. C. (2009). How does transcranial magnetic stimulation modify neuronal activity in the brain? Implications for studies of cognition. Cortex 45, 1035-1042.

Sparenberg, P., Springer, A., and Prinz, W. (2012). Predicting others' actions: evidence for a constant time delay in action simulation. Psychol. Res. 76, 41-49.

Springer, A., and Prinz, W. (2010). Action semantics modulate action prediction. Q. J. Exp. Psychol. 63, 2141-2158.

Stadler, W., Schubotz, R. I., von Cramon, D. Y., Springer, A., Graf, M., and Prinz, W. (2011). Predicting and memorizing observed action: differential premotor cortex involvement. Hum. Brain Mapp. 32, 677-687.

Sternberg, S., Monsell, S., Knoll, R. L., and Wright, C. E. (1978). "The latency and duration of rapid movement sequences: comparisons of speech and typewriting," in Information Processing in Motor Control and Learning, ed. G. E. Stelmach (New York: Academic Press), 117-152.

Talairach, J., and Tournoux, P. (1988). Co-Planar Stereotaxic Atlas of the Human Brain. New York: Thieme.

Tanaka, S., Honda, M., and Sadato, N. (2005). Modality-specific cognitive function of medial and lateral human Brodmann area 6. J. Neurosci. 25, 496-501.

Terao, Y., Ugawa, Y., Suzuki, M. Sakai, K., Hanajima, R., GembaShimizu, K., and Kanazawa, I. (1997). Shortening of simple reaction time by peripheral electrical and submotor-threshold magnetic cortical stimulation. Exp. Brain Res. 115, 541-545.

Tomassini, V., Jbabdi, S., Klein, J. C., Behrens, T. E., Pozzilli, C., Matthews, P. M., Rushworth, M. F., and Johansen-Berg, $\mathrm{H}$. (2007). Diffusion-weighted imaging tractography-based parcellation of the human lateral premotor cortex identifies dorsal and ventral subregions with anatomical and functional specializations. J. Neurosci. 27, 10259-10269.

Umiltà, M. A., Kohler, E., Gallese, V., Fogassi, L., Fadiga, L., Keysers, C., and Rizzolatti, G. (2001). I know what you are doing. A neurophysiological study. Neuron 31, 155-165.

Urgesi, C., Candidi, M., Ionta, S., and Aglioti, S. M. (2007a). Representation of body identity and body actions in extrastriate body area and ventral premotor cortex. Nat. Neurosci. 10, 30-31.

Urgesi, C., Calvo-Merino, B., Haggard, P., and Aglioti, S. M. (2007b). Transcranial magnetic stimulation reveals two cortical pathways for visual body processing. J. Neurosci. 27, 8023-8030.

Urgesi, C., Maieron, M., Avenanti, A. Tidoni, E., Fabbro, F., and Aglioti, S. M. (2010). Simulating the future of actions in the human corticospinal system. Cereb. Cortex 20 , 2511-2521.

Wassermann, E. M. (1998). Risk and safety of repetitive transcranial magnetic stimulation: report and suggested guidelines from the International Workshop on the Safety of Repetitive Transcranial Magnetic Stimulation, June 5-7, 1996. Electroencephalogr. Clin. Neurophysiol. 108, 1-16.

Wilson, M., and Knoblich, G. (2005). The case for motor involvement in perceiving conspecifics. Psychol. Bull. 131, 460-473.
Windischberger, C., Lamm, C., Bauer, H., and Moser, E. (2003). Human motor cortex activity during mental rotation. Neuroimage 20, 225-232.

Wise, S. P., Boussaoud, D., Johnson, P. B., and Caminiti, R. (1997). Premotor and parietal cortex: corticocortical connectivity and combinatorial computations. Annu. Rev. Neurosci. 20, 25-42.

Conflict of Interest Statement: The authors declare that the research was conducted in the absence of any commercial or financial relationships that could be construed as a potential conflict of interest.

Received: 25 August 2011; accepted: 02 February 2012; published online: $20 \mathrm{Feb}$ ruary 2012.

Citation: Stadler W, Ott DVM, Springer A, Schubotz RI, Schütz-Bosbach S and Prinz $W$ (2012) Repetitive TMS suggests a role of the human dorsal premotor cortex in action prediction. Front. Hum. Neurosci. 6:20. doi 10.3389/fnhum.2012.00020

Copyright () 2012 Stadler, Ott, Springer, Schubotz, Schütz-Bosbach and Prinz. This is an open-access article distributed under the terms of the Creative Commons Attribution Non Commercial License, which permits non-commercial use, distribution, and reproduction in other forums, provided the original authors and source are credited. 